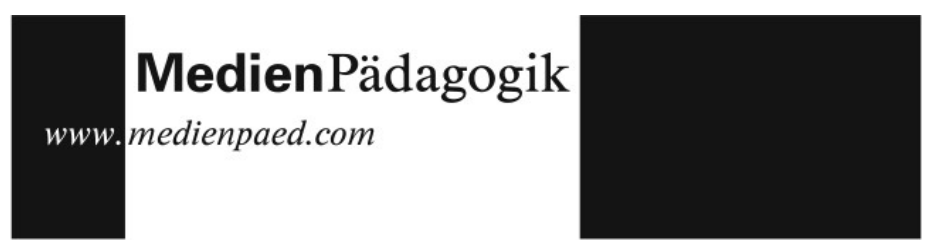

\title{
Rezensionen
}

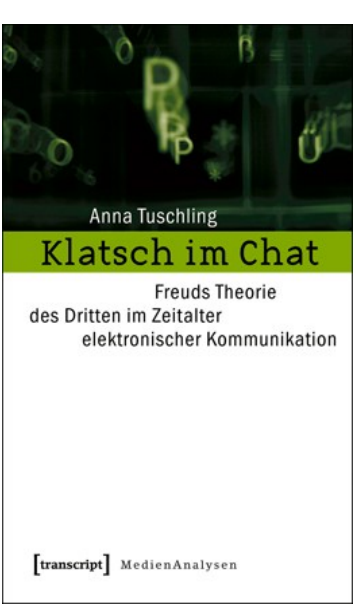

Anna Tuschling

Klatsch im Chat

Freuds Theorie des Dritten um Zeitalter elektronischer Kommunikation

Bielefeld: transcript, 2009. 294 Seiten

ISBN: 978-3-89942-952-

$€ 28,80$, CHF 36,00

\section{Chatten - geklatscht wird auch hier!}

Das Internet ist zu einem Medium avanciert, das für viele Menschen aus dem privaten wie auch beruflichen Alltag nicht mehr wegzudenken ist. Im besonderen Maße gilt dies gewiss für seine kommunikativen Offerten. Eine beliebte Art, im neuen Kulturraum zu kommunizieren ist das Chatten. Es bezeichnet eine Form der computervermittelten Kommunikation im Internet, die dadurch charakterisiert ist, dass der Gesprächsverlauf nahezu synchron verläuft und die anwesenden Teilnehmer/innen sich ohne spürbare zeitliche Verzögerung verständigen können.

Mit ihrem Buch Klatsch im Chat möchte Anna Tuschling, Juniorprofessorin für Medien und anthropologisches Wissen an der Ruhr-Universität Bochum, das Chatten nun als «ein mediengeschichtliches und dabei pragmatisches Übergangsphänomen in der Entwicklung des Internet» (11) in den Blick nehmen, «an dem sich die elektronische Artikulation des Symbolischen» (ebd.) aus ihrer Sicht besonders deutlich veranschauliche. Das erklärte Ziel der theoretisch und empirisch ausgerichteten Studie ist es, «die Matrix und medialen Arrangements, welche Chatroom genannt werden» (12) zu analysieren und «Möglichkeitsbedingungen und Voraussetzungen elektronischer Textverständigung» (ebd.) zu explorieren. Dabei wählt die 


\section{Rezensionen}

Autorin einen Ansatz, der sich grundlegend von bisherigen Unternehmungen der Chat-Forschung unterscheidet.

Im ersten Kapitel ihres Buches führt die Autorin ausführlich und dezidiert in das Thema Chat ein. Neben der Entwicklung und den Spezifika dieser Kommunikationsform setzt sich Tuschling kritisch mit dem Stand der Forschung auseinander und beleuchtet Mythen und Vorbehalte gegenüber einer medialen Kommunikationspraxis, die anders als die E-Mail, immer wieder Anlass kulturkritischer Auseinandersetzungen ist.

Mit dem zweiten Kapitel schlägt Tuschling den Bogen zu jener Theorie, die ihr als Schlüssel zum Phänomen des Chatten dient: Siegmund Freuds «Theorie des Dritten». Dabei strebt sie keine Gesamtschau des komplexen theoretischen Gedankengebäudes an. Vielmehr konzentriert sie sich auf Freuds Reflexionen, in der «Gesprächsdialoge strukturell als Triaden» (35) behandelt werden. Die Autorin setzt sich hier zunächst dezidiert mit Arbeiten auseinander, die der Traumdeutung und der Konstitution des Witzes gewidmet sind. Im Sinne Freuds verbinde sich mit der Figur des Dritten die entscheidende Frage, aus welchem Grund und mit welcher Absicht eine Person einen Witz erzähle. So könne der Erzähler eines Witzes gemeinhin nicht ohne die Existenz eines Dritten über seinen Witz lachen. Jener Vorgang setze stets eine Triade, bestehend aus dem Erzähler, dem Witz bzw. Witzobjekt und schließlich dem Zuhörer, voraus. An diesen Grundgedanken anschließend fokussiert Tuschling sodann auf Witz, Klatsch und Chat als spezifische Ausdrucksformen triadischer Gesprächskonstellationen. Der Witz ermögliche Anerkennung, dem Klatsch liege eine inverse Anerkennung vor und der Chat lasse sich «als Suche nach der Möglichkeitsbedingung von Anerkennung bezeichnen» (ebd.). Augenscheinlich tritt hier die Verbindung zum Klatsch im Chat zutage. Im Rückgriff auf Freuds enigmatische Theorie des Dritten sieht Tuschling einen Weg, Chat-Strukturen als Klatschtriaden zu decodieren.

Im dritten Kapitel vollzieht die Autorin endgültig den Schritt zum Untersuchungsgegenstand. Dabei wendet sie Freuds Erkenntnisse systematisch auf das Phänomen des Chattens an - «Chatprotokolle wie 


\section{Rezensionen}

Witze zu lesen» (186). Denn wenn auch ein «explosives Gelächter» (121), als welche sich die Figur des Dritten bei Freud darstelle, beim Klatsch fehle, seien Witz und Klatsch «nicht nur strukturell verwandt», sondern diese gingen «ineinander über» (123). Den Gemeinsamkeiten von Witz und Klatsch widmet sich die Autorin recht ausführlich, um auf dieser Grundlage, die Wesenszüge ihres eigenen Forschungsgegenstandes weiter zu elaborieren.

Nach den so entfalteten Bestimmungen, erarbeitet Tuschling im vierten Kapitel die Spezifika des digitalen Klatsches - dem Chatten. In einen Chatroom, so ihre Annahme, kämen die Teilnehmenden wegen eines «zweck- und zwanglosen Chats, der Plauderei und des Klatsches» (155) zusammen. Die Analyse der Autorin rückt das «zweckoffene Experimentieren mit Zeichen, Schrift und medialer Anordnung als Charakteristika des Chattens» (ebd.) entsprechend in den Mittelpunkt. Dabei widmet sie sich zunächst den Spezifika der Kommunikation im Chat (u.a. Nickname, Mühlenprinzip, Akronymen/Emoticons, Flaming) und bündelt anhand beispielhafter Sequenzen «drei typische Konstellationen und Hauptausprägungen des Klatsches im Chat» (187): «Klatsch vertreibt die angegriffenen Teilnehmer, Klatsch wendet sich gegen die Klatschenden, Klatsch ereignet sich in Abwesenheit des Gesprächsobjektes» (ebd.).

Tulschings Buch ist eine komplexe und überzeugende Arbeit, die sich auf eine originelle Weise dem Phänomen des Chattens nähert. Das Dissertationsprojekt, das sich stark von der bisherigen Chatforschung unterscheidet und sich daher auch nicht einfach in die bestehende Forschungslinien einreiht, entfaltet einen neuen und interessanten Blickwinkel auf ein noch wenig erkundetes Forschungsfeld. Wünschenswert, aufgrund der Komplexität der Arbeit, wären Zusammenfassungen am Ende der einzelnen Kapitel gewesen. 